\title{
Flaps for Oral and Maxillofacial Reconstruction: Review of Literature and a Clinical Guide to the Clinicians
}

\author{
Gaurav Mittal' ${ }^{1}$ Anmol Agarwal' Gaurav Kataria ${ }^{1}$ \\ ${ }^{1}$ Department of Oral and Maxillofacial Surgery, Institute of Dental \\ Studies and Technologies, Modinagar, Uttar Pradesh, India
}

Address for correspondence Gaurav Mittal, MDS, 1612, Tower 3C, Golf Links, Landcraft, NH 24, Ghaziabad 201002, Uttar Pradesh, India (e-mail: drgauravmittal@rediffmail.com).

\begin{abstract}
Keywords

- distant flap

- free flap

- local flap

- maxillofacial

- reconstruction

There are many different ways documented for reconstruction of oral and maxillofacial (OMF) region among which the use of flaps (local or distant) is commonly practiced worldwide. Modern techniques of OMF reconstruction aim to restore function as well as improve aesthetics. This article intends to review the literature on varied flaps used in oral and maxillofacial surgery (OMFS) and summarize their precise clinical implications taking into consideration the ease, difficulties, and the ultimate clinical outcomes. A review of the literature of local or distant flaps used in OMFS was done using Google database. There are many different methods of reconstruction in patients who have had defect in the OMF region due to vascular reasons. It is important for the OMF surgeon who is involved with the management of such patients to have an understanding regarding the choice of flap used to reconstruct such defects.
\end{abstract}

\section{Introduction}

Management of varied pathologic lesions of the jaws often demands resection in partial or total along with the surrounding structures, producing defects of varying sizes. Such defects may also result due to maxillofacial trauma, burns, etc. Reconstruction of such defects (-Table $\mathbf{1}$ ) is important in many respects that include restoration of function and aesthetics rehabilitation.

The first and the foremost technique that is thought for the reconstruction of maxillofacial defects is the use of soft tissue flaps that range from different sizes and forms (-Table 2). ${ }^{1,2}$ These flaps may comprise simple advancements of skin to composites involving many different types of tissue. This article aims to review the literature on plethora of flaps used in oral and maxillofacial surgery (OMFS) and summarize their precise clinical implications taking into consideration the ease, difficulties, and the ultimate clinical outcomes.

\section{Terminologies and General Concepts}

The term flap originated in the 16th century from the Dutch word "flappe," meaning something that hung broad and loose, fastened only by one side. The history of flap dates as far back as 600 BC, when Sushruta Samita described nasal reconstruction using a check flap.

A flap in technical terms is a tissue that is transferred from one side (donor site) to another (recipient site) while maintaining its own blood supply. Depending on the position of the donor and recipient sites, flaps can be broadly classified as local or distant (regional) flaps.

Local flaps are those that are derived from the immediate area of resection common examples of these include buccal pad of fat flap, nasolabial flap, and facial artery musculomucosal (FAMM) flap. These types of flap are advanced, transposed, or rotated into position and a supplied by either an axial pattern or by a random pattern. Axial flaps receive their blood supply from a single nutrient vessel whereas random pattern flaps receive capillary blood supply in a random pattern from all direction and not from a single nutrient vessel. The Rehrmann buccal advancement flap is good example of a random flap.

In distant or regional flaps the donor site is distant from the recipient site. These flaps were used to be the workhorse of reconstruction of the oral cavity in the past, but they are now losing their shine due to introduction of better options. 
Table 1 Indications for reconstruction using flaps

\begin{tabular}{|l|}
\hline Defects produced by cancer surgeries \\
\hline Posttraumatic defects \\
\hline Facial reanimation \\
\hline Oro-antral communication \\
\hline Palatoplasties \\
\hline Cleft palates \\
\hline Postburn defects \\
\hline Major pathologies, viz. cysts, etc. \\
\hline Hair transplantation \\
\hline Rhinoplasties \\
\hline
\end{tabular}

Table 2 Classification of flaps based on blood supply, including the Mathes and Nahai subclassification

\begin{tabular}{|l|}
\hline Random (no named blood vessel) \\
\hline Axial (named blood vessel) Mathes and Nahai classification \\
\hline One vascular pedicle (e.g., tensor fascia lata) \\
\hline Dominant pedicle(s) and minor pedicle(s) (e.g., gracilis) \\
\hline Two dominant pedicles (e.g., gluteus maximus) \\
\hline Segmental vascular pedicles (e.g., sartorius) \\
\hline
\end{tabular}

These flaps have an intact vascular pedicle where the blood supply originates from a bridging segment connection the pedicle to the flap. The bridging segment is normally covered by skin and subcutaneous tissue. If this skin is removed, the flap becomes and island flap. Examples of regional flaps include pectoralis major flap and temporalis flap.

Free vascularized tissue transfer or free flaps is a modification of regional flap where flap is harvested from a distant site by dividing its vascular pedicle. This pedicle is subsequently anastomosed to the blood supply and drainage at the site of the newly created defect. Tissues that can be transferred as a flap includes skin, fascia, bone, and bowel. Combination of different tissues are referred to as composite free flaps examples osteocutaneous free flaps, etc.

Compared with skin grafts, local flaps often produce superior functional and esthetic results. A great advantage of local tissue transfer is the close resemblance to the missing skin in color and texture. Regional tissue can also be recruited to repair facial defects with certain limitations. ${ }^{3}$ After the advent of free flaps, clinicians have overcome the difficulties of other flaps, but at the same time, free flaps are a sensitive technique and attract surgical expertise.

\section{Discussion}

Defects as a result of tumor resection in the OMF region may leave the patient with a significant functional and esthetic defect that is clearly related to the anatomic site and tumor size. A plethora of regional cutaneous and myocutaneous flaps are available for the management of such defects and are well-accepted surgical option. ${ }^{4}$

In OMFS, reconstruction of surgical defects due to pathological lesions or tumors has always been a challenge for clinicians due to its anatomical complexity that makes the reconstruction of these defects a tedious task.

In the past, most oropharyngeal defects were closed primarily using skin flaps or tubed pedicle flaps of skin from the trunk. These included forehead flap, deltopectoral flap, pectoralis major myocutaneous flap (PMMC), etc. However, defects reconstructed using regional pedicle flaps gave a compromised esthetic and functional result, and there arose the need for better options. Researchers have worked upon multiple newer techniques, but the fact that each of them has got certain limitations has left the clinician with a lot of disparity and personal choice ( - Table 3 ).

\section{Local Flaps}

\section{Buccal Fat Pad}

Buccal fat pad (BFD) is an encapsulated, specialized fatty tissue located between the buccinator muscle medially, the anterior margin of the masseter, and the mandibular ramus and zygomatic arch laterally ( - Fig. 1). BFD was known as a surgical difficulty for many years because of its accidental encounter during various surgical procedures in the pterygomandibular area but later Egyedi ${ }^{4}$ has proven it as a boon for OMF surgeons. The flap is harvested through an incision in the posterosuperior vestibular sulcus opposing the second molar tooth. After incising the fascia, the fat pad is easily delivered into the oral cavity by blunt dissection. If the defect is not continuous with the donor site, the flap can be tunneled through mucosa. The flap is sutured into position and allowed to mucosalize, which occurs within 3 to 4 weeks. ${ }^{5}$ This is a pedicled flap with its blood supply derived from the buccal and deep temporal branches of the maxillary artery and from vessels from the transverse facial artery. Because of its accessibility, available size, and mini mal donor site morbidity, it has been used reliably to reconstruct soft and hard palatal, retro molar fossa, buccal mucosa, and oropharyngeal defects. The flap is also used to help repair oral antral communications, in conjunction with an advancement flap, caused by dental extraction. ${ }^{6}$

\section{Facial Artery Musculomucosal Flap}

FAMM flap was described by Pribaz and colleagues in 1992, ${ }^{7}$ which contains mucosa, submucosa, buccinator muscle, buccal fat, and facial artery. It has been used in oropharyngeal reconstruction following cancer ablation, congenital

Table 3 Criteria for choosing a an ideal flap

Provision of a suitable color match to the surrounding skin of the recipient bed

Assurance of a compatible thickness

Retention, or provision of recovery, of clinically perceptible sensory innervations

Attainment of sufficient laxity and tissue ablation such that mobile margins, as in an eyelid or lip, are spared retraction and deranged function

Assurance that the resultant suture lines of either primary or secondary defects are restricted to anatomic units and fall within natural skin lines 
or posttraumatic oroantral fistulas, cleft palate defects, for soft palate lengthening, and to repair defects of the septal mucosa, alveolar ridge, floor of mouth, vermilion, and orbits. ${ }^{8}$ The flap can be either superiorly or inferiorly based as it can be used either in the anterior maxilla region, lips, or anterior floor of mouth (-Fig. 2). It has been shown to be a useful flap in certain situations although the variability of the facial artery can make it less reliable. ${ }^{9}$

\section{Nasolabial Flaps}

Hagan and Walker formally described the nasolabial musculocutaneous flap in $1988 .{ }^{10}$ Garatea described a modification of the musculocutaneous nasolabial flap for intraoral reconstruction in $1991 .{ }^{11} \mathrm{~A}$ nasolabial flap consists of a finger or tissue lying either side of the nasolabial skin crease. It can be based superiorly, inferiorly, or tunneled into the oral cavity, and is mainly used as a transposed flap to reconstruct defects of the side of the nose, upper lip, lower lip, and cavity (-Fig. 3). This flap is a cutaneous flap based on the facial artery. As they utilize the nasolabial folds, the scar associated with the flap is minimal. These flaps are placed in position,

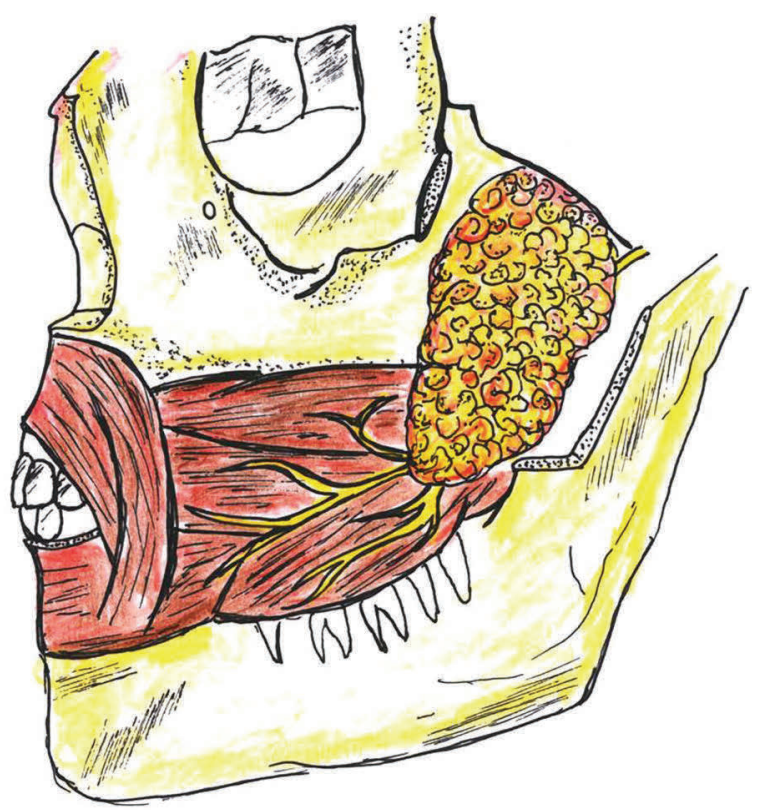

Fig. 1 Buccal pad of fat flap.
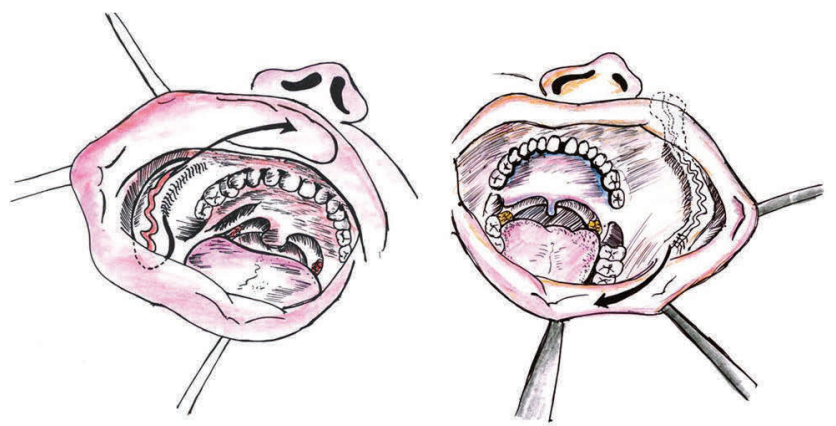

Fig. 2 Facial artery musculomucosal flap.

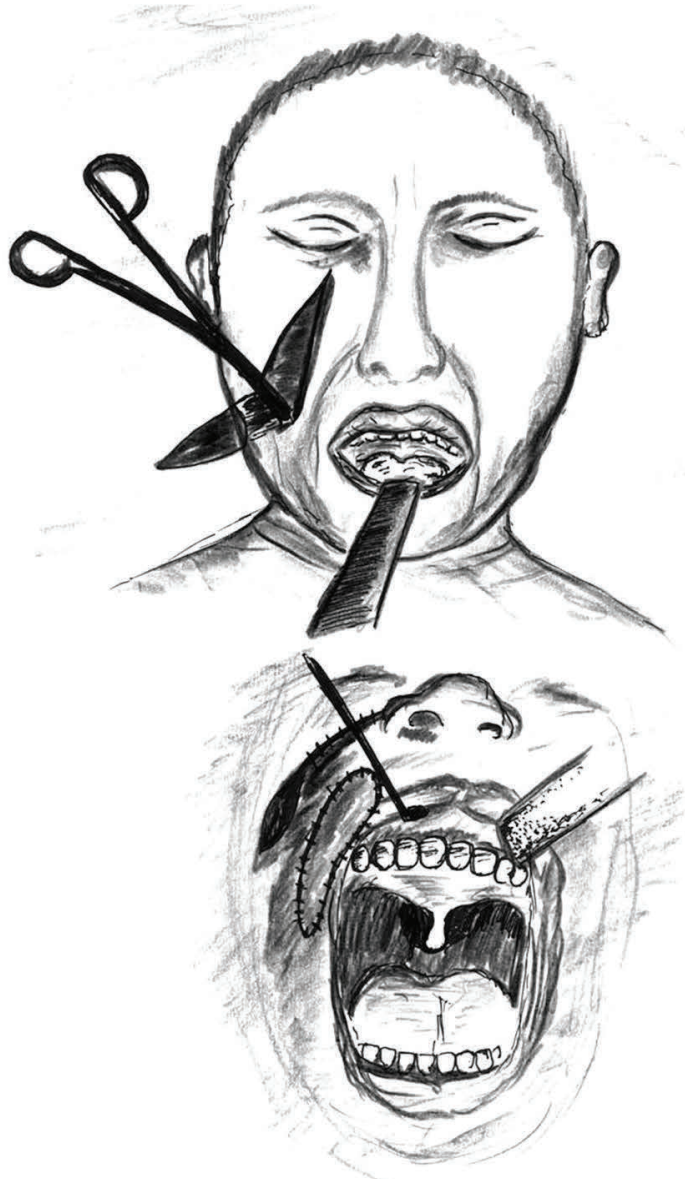

Fig. 3 Nasolabial flap.

and the pedicle is then released 4 weeks later as a secondary procedure once the flap has gained a blood supply from the recipient bed. As per the recent advances, tunneling of the flap into the oral cavity eliminates the need of pedicle release.

\section{Regional Flaps}

\section{Pectoralis Major Myocutaneous Flap}

Pectoralis major myocutaneous flap is the most commonly used muscle skin transfer used in soft tissue reconstruction of defects of the upper neck and jaw region that may include the underlying ribs. It was first described by Hueston and McConchie in 1968. Pectoral is major flap was introduced into head and neck reconstruction by Ariyan in 1979.11,12 PMMC flap has an axial blood supply and is based superiorly on the pectoral branch of the thoracoacromial artery. It is very useful in the head and neck region for reconstruction of soft tissue defects of the oropharynx, oral cavity, hypopharynx, and skin of the neck, to augment pharyngeal repairs following salvage. It is tunneled underneath the skin of the neck and so can re-create the sternocleidomastoid and protect the carotid vessels ( $\boldsymbol{- \text { Fig. }}$ 4). It is a well-accepted versatile and reliable reconstructive technique for the head and neck region. However, because of its bulk, especially in the obese, the esthetic outcome is poor. Still it can be used as an option when free flaps are not indicated and shows failure. 


\section{Temporalis Flap}

This flap was first described in $1995^{6}$ and has been used for reconstruction of temporomandibular joint and orbitalzygomatic defects. It is supplied by the deep temporal branches of the maxillary artery can be harvested with cranial bone or coronoid process of the mandible (-Fig. 5). It has also been used as a myofascial flap intraorally. Estellés Ferriol et al in $2005^{13}$ discussed that the myofascial flap is simpler to manage than the free microvascular flaps and safe because of

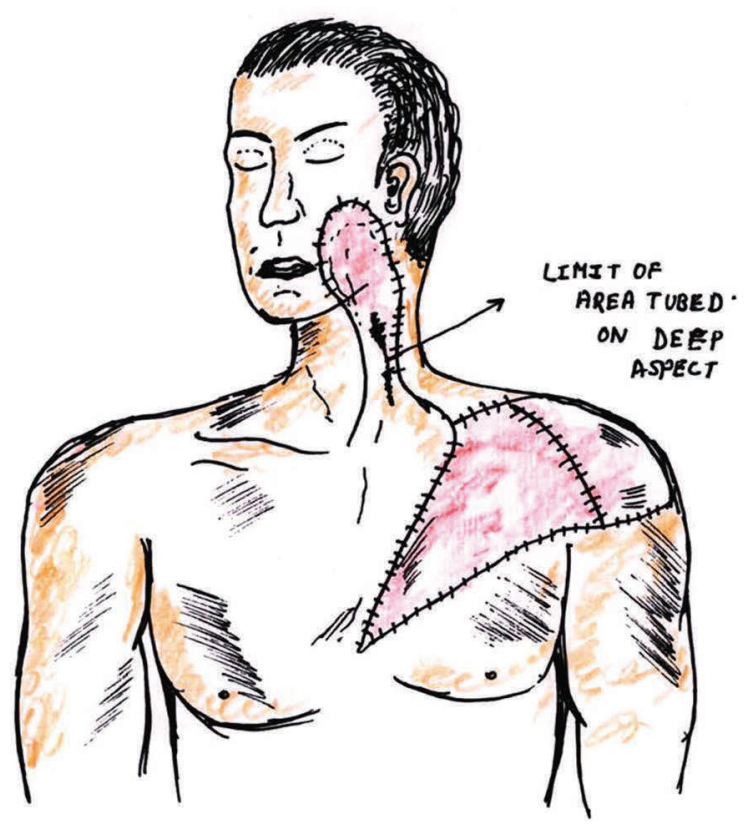

Fig. 4 Pectoralis major myocutaneous flap.

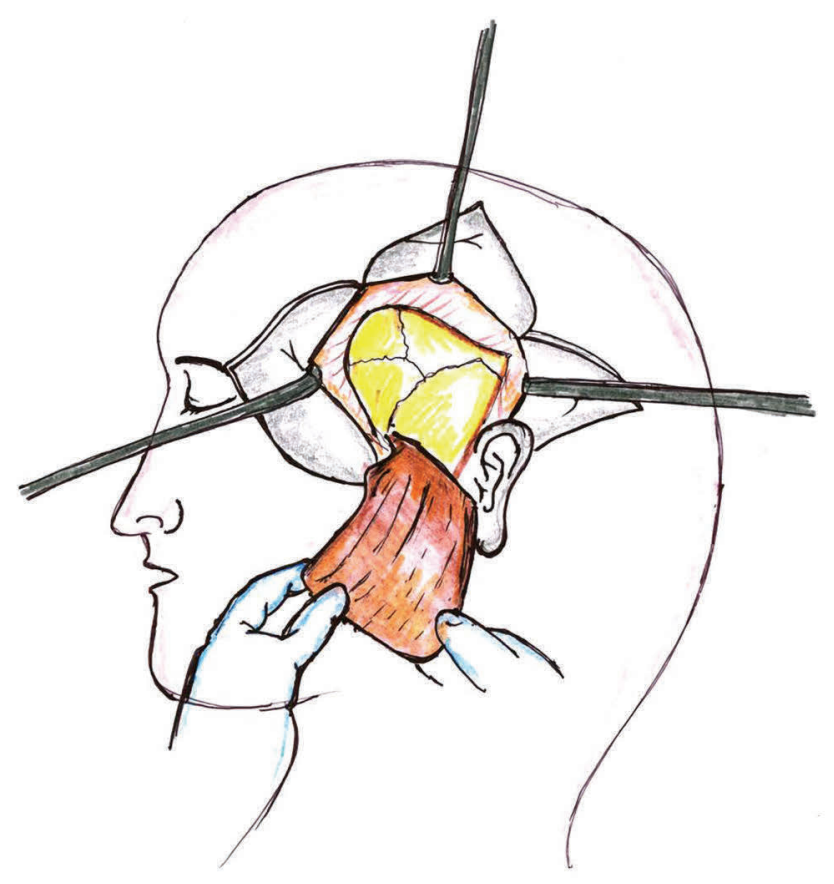

Fig. 5 Temporalis flap. its great vascularization. The merits of this flap includes its slim size, flexibility, and the fascia in contract with the oral cavity, which epithelize within 3 weeks making it resistant to the proteolytic action of saliva. The flap is capable of supporting skin grafts and/or nourishing bone grafts. ${ }^{14}$ It requires only one surgical site, and the incision is extended into the hairline, and the muscle is elevated from an inconspicuous area. Thus it produces minimal functions and esthetic morbidity. Temporalis muscle flap has been criticized because of its short arc of ration due to which it cannot reach the midline and most of the muscle bulk is used in the pedicle.

\section{Free Flaps}

\section{Radial Forearm Free Flap}

Radial forearm free flap was first described by Yang et al in $1981^{14}$ and popularized by Soutar et al. ${ }^{15}$ It is the workhorse of oral reconstruction due to its versatility, reliability, and flexibility. It is most often harvested as a fasciocutaneous flap that undergoes epithelialization and submucosal fibrosis providing the ideal attached mucosal lining for prosthetic rehabilitation ( - Fig. 6). It is excellent for reconstruction of oral cavity, oropharyngeal, and hypopharyngeal defects, such as those found after hemiglossectomy or laryngectomy with partial pharyngectomy. Advantages with this flap include the large amount of thin, pliable skin that is available, relatively easy to raise, and has reliable vasculature, but some tissue includes the need for a split-thickness skin graft and the unsatisfactory appearance of the forearm scar.

\section{Fibular Free Flap}

Fibular free flap (FFF) was first used for mandible reconstruction by Hidalgo in $1989 .{ }^{16}$ It is the most commonly used free flap for mandible reconstruction in this era. The vascular pedicle includes the personal artery and vein. FFF is popular for several reasons; for example, it can be harvested by a second surgical team and provides a long segment of the bone $(\sim 25 \mathrm{~cm})$ that is generally not a weight-bearing bone of the body and that can actually be cut into smaller segments to re-create curvature of the mandible ( - Fig. 7). Osseointegrated dental implants can often be placed postoperatively. ${ }^{5}$ Even distraction osteogenesis appliances have been successfully applied to and lengthened FFFs. It can also be used for bony reconstruction of the maxilla and orbital floor. If the patient's blood supply to the foot is dominated by the peroneal artery, an FFF from that leg is contraindicated. ${ }^{5}$

\section{Free Iliac Crest Flap}

This osteocutaneous flap uses the iliac crest bone with or without skin based on the deep circumflex iliac artery (DCIA) and vein. This flap was first described for reconstruction of the maxillofacial region by Bitter. ${ }^{17}$ The main benefit of using the iliac crest as a free flap is the curvature of the ilium that is similar to that of the mandible ( $\boldsymbol{- \text { Fig. }}$ 8). It can be quite a morbid procedure. Though causes gait problems due to pain, abdominal wall weakness, and frank herniation, some of these problems may be resolved by only using the inner table of the ilium. There may also be a considerable amount 

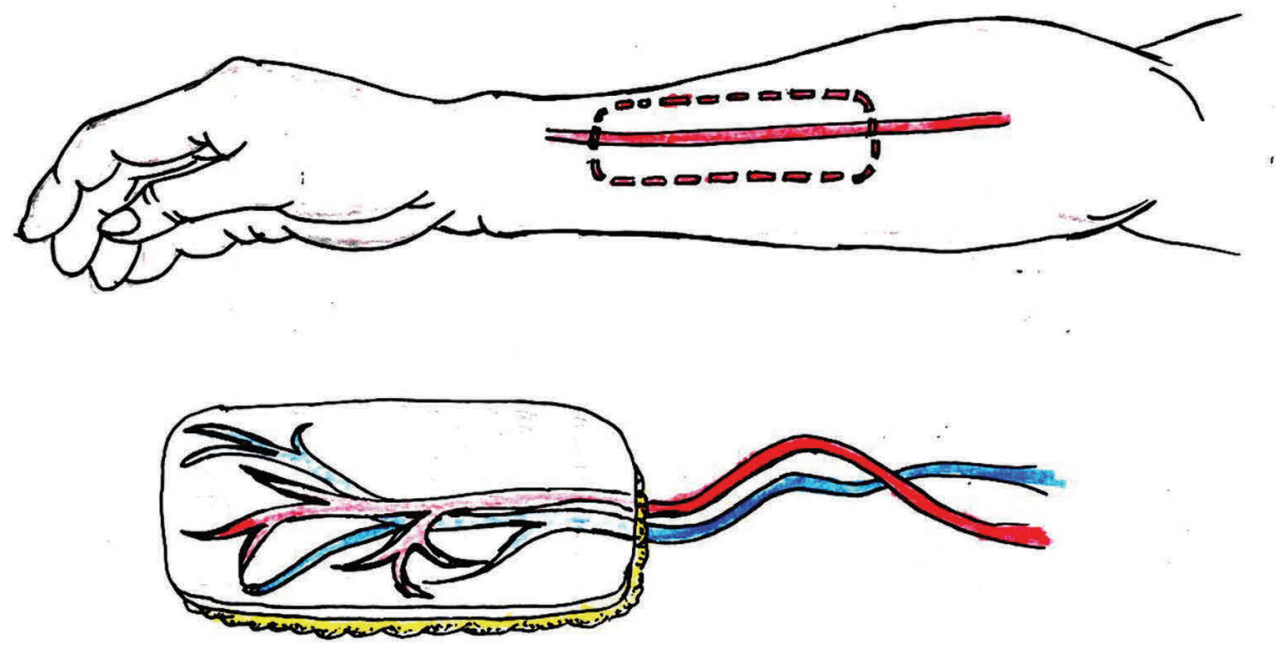

Fig. 6 Free radical forearm flap.

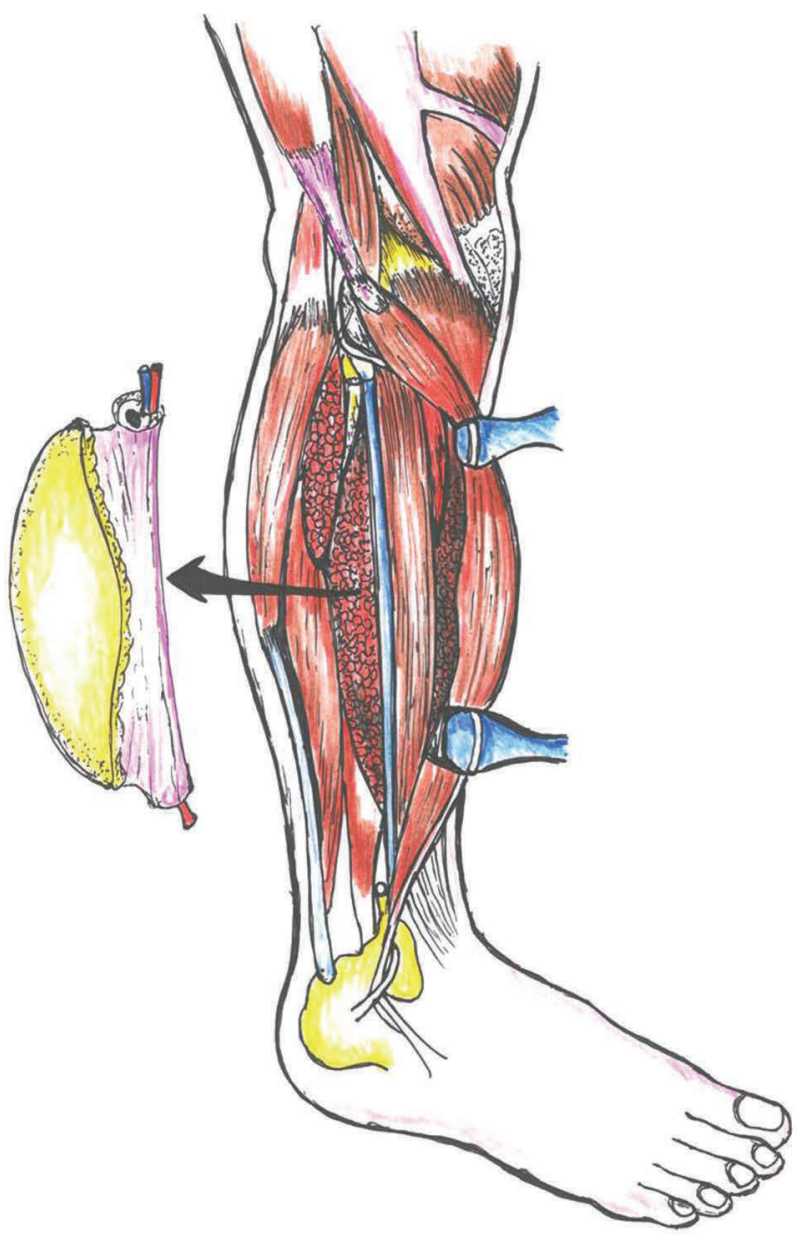

Fig. 7 Free fibula flap.

of soft tissue associated with the blood supply for the free flap, making it bulky and susceptible to trauma from upper teeth and making prosthetic rehabilitation very difficult. ${ }^{5}$

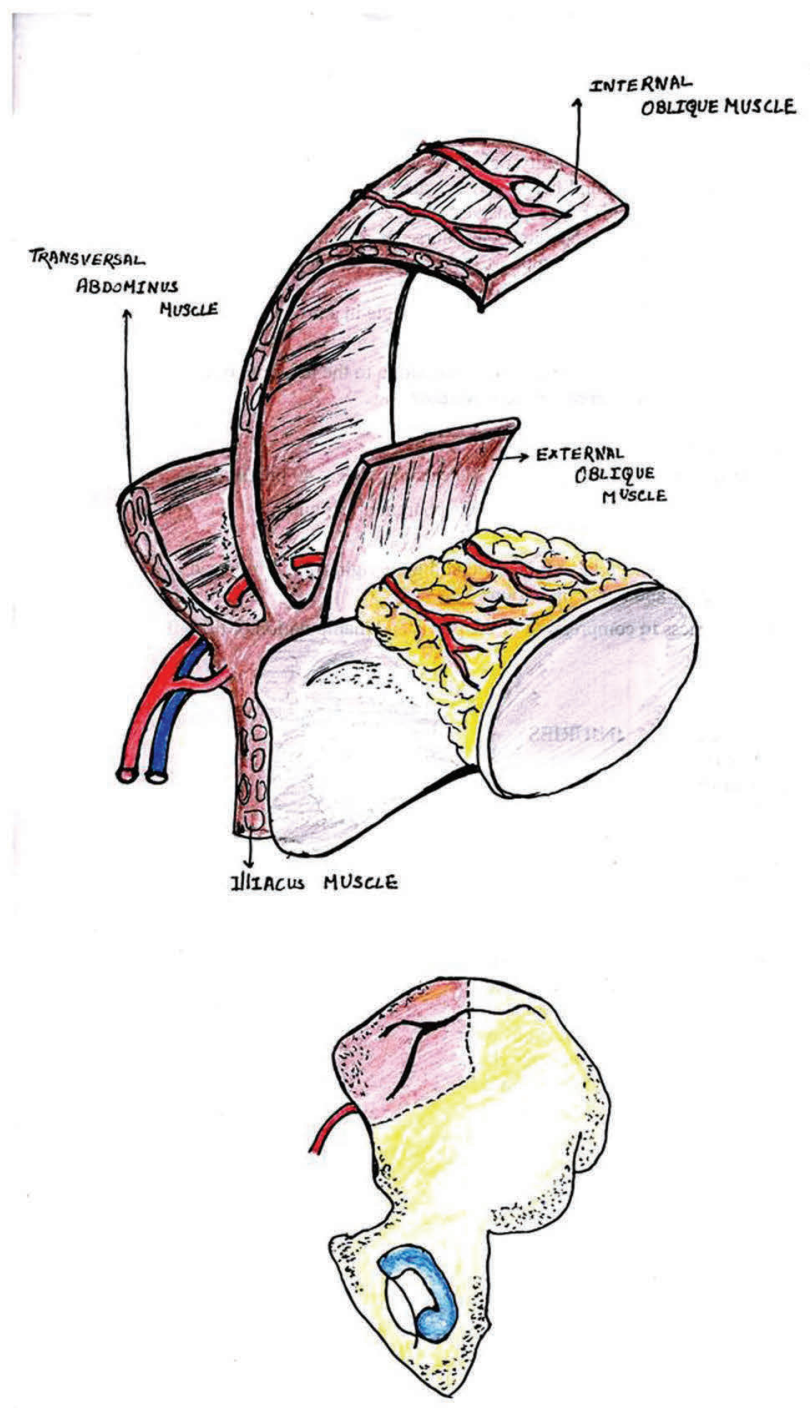

Fig. 8 Free iliac crest flap. 


\section{Conclusion}

There is a wide variety of reconstructive techniques available for use in the OMF region post-ablative surgery. Smaller defects can be repaired with grafts such as split grafts whereas bigger defects can be dealt with local and regional flaps such as BFD, FAMM flap, temporalis flap, PMMC flap, etc. In this era, majority of larger defects are repaired using free vascularized tissue transfer, particularly the radial forearm free flap and FFF.

These flaps may be used on their own or in combination with other flaps to close a variety of three-dimensional defects that may occur as a result of tumor resection. It is important for the OMF surgeon to become familiar with these techniques, particularly those of free flap reconstructive. Familiarity with common problems associated with cutaneous free flap reconstruction such as the retention and growth of hair and excessive flap thickness is also important. Such knowledge will help in understanding the problems of reconstruction and aid planning the oral rehabilitation of the patient post convalescence.

\section{Funding}

None.

\section{Conflict of Interest}

None declared.

\section{References}

1 McGregor IA, McGregor AD. Fundamental Technique of Plastic Surgery and Their Surgical Application. 9th ed. London: Churchill Livingstone; 1995

2 Herford AS, Ghali GE. Peterson's Principles of Oral and Maxillofacial Surgery. 2nd ed. London, UK: BC Decker; 2004:769-782

3 Eckardt AM, Kokemüller H, Tavassol F, Gellrich NC. Reconstruction of oral mucosal defects using the nasolabial flap: clinical experience with 22 patients. Head Neck Oncol 2011;3:28
4 Egyedi P. Utilization of the buccal fat pad for closure of oro-antral and/or oro-nasal communications. J Maxillofac Surg 1977;5(4):241-244

5 Snyder MC, Gutowski KA. Head and neck: reconstruction. Sel Read Plast Surg 2004;10:4

6 Chandu A, Bridgeman AM, Smith AC, Flood SJ. Reconstructive techniques for the repair of oral and maxillofacial oncological procedures: what are they, how do they work and what do they look like? Aust Dent J 2002;47(2):99-105

7 Pribaz JJ, Meara JG, Wright S, Smith JD, Stephens W, Breuing KH. Lip and vermilion reconstruction with the facial artery musculomucosal flap. Plast Reconstr Surg 2000;105(3):864-872

8 Dupoirieux L, Plane L, Gard C, Penneau M. Anatomical basis and results of the facial artery musculomucosal flap for oral reconstruction. Br J Oral Maxillofac Surg 1999;37(1):25-28

9 Schmidt BL, Dierks EJ. The nasolabial flap. Oral Maxillofac Surg Clin North Am 2003;15(4):487-495-v

10 Hagan WE, Walker LB. The nasolabial musculocutaneous flap: clinical and anatomical correlations. Laryngoscope 1988;98(3):341-346

11 Ariyan S. The pectoralis major myocutaneous flap. A versatile flap for reconstruction in the head and neck. Plast Reconstr Surg 1979;63(1):73-81

12 Hueston JT, McConchie IH. A compound pectoral flap. Aust N Z J Surg 1968;38(1):61-63

13 Estellés Ferriol JE, Carrasco Llatas M, Ferrer Ramírez MJ, López Mollá C, Baviera Granel N, Dalmau Galofre J. [Temporalis myofascial flap: technique description and results in our patients] [in Spanish]. Acta Otorrinolaringol Esp 2005;56(6):257-260

14 Yang G, Chem B, Gao Y, et al. Forearm free skin flap transplantation. Zhonghua Yi Xue Za Zhi 1981;61:139

15 Soutar DS, Scheker LR, Tanner NSB, McGregor IA. The radial forearm flap: a versatile method for intra-oral reconstruction. Br J Plast Surg 1983;36(1):1-8

16 Hidalgo DA. Fibula free flap: a new method of mandible reconstruction. Plast Reconstr Surg 1989;84(1):71-79

17 Bitter K. Bone transplants from the Iliac crest to the maxillo-facial region by the microsurgical technique. J Maxillofac Surg 1980;8(3):210-216 